\title{
Physiological noise in human cerebellar fMRI
}

\author{
Wietske van der Zwaag ${ }^{1} \cdot$ João Jorge $^{1,2} \cdot$ Denis Butticaz ${ }^{1} \cdot$ Rolf Gruetter $^{1,3}$
}

Received: 19 November 2014 / Revised: 19 March 2015 / Accepted: 25 March 2015 / Published online: 18 April 2015

(C) ESMRMB 2015

\begin{abstract}
Objectives To compare physiological noise contributions in cerebellar and cerebral regions of interest in high-resolution functional magnetic resonance imaging (fMRI) data acquired at $7 \mathrm{~T}$, to estimate the need for physiological noise removal in cerebellar fMRI.

Materials and methods Signal fluctuations in high resolution (1 mm isotropic) 7T fMRI data were attributed to one of the following categories: task-induced BOLD changes, slow drift, signal changes correlated with the cardiac and respiratory cycles, signal changes related to the cardiac rate and respiratory volume per unit of time or other. $R_{\text {adj }}^{2}$ values for all categories were compared across regions of interest.

Results In this high-resolution data, signal fluctuations related to the phase of the cardiac cycle and cardiac rate were shown to be significant, but comparable between cerebellar and cerebral regions of interest. However, respiratory related signal fluctuations were increased in the cerebellar regions, with explained variances that were up to $80 \%$ higher than for the primary motor cortex region.

Conclusion Even at a millimetre spatial resolution, significant correlations with both cardiac and respiratory RETROICOR components were found in all healthy volunteer data. Therefore, physiological noise correction is highly
\end{abstract}

Wietske van der Zwaag

Wietske.vanderzwaag@epfl.ch

1 Centre d'Imagerie Biomédicale (CIBM), Station 6, École Polytechnique Fédérale de Lausanne, 1015 Lausanne, Switzerland

2 Instituto Superior Técnico, Lisbon, Portugal

3 Department of Radiology, Université de Lausanne, Lausanne, Switzerland likely to improve the temporal signal-to-noise ratio (SNR) for cerebellar fMRI at 7T, even at high spatial resolution.

Keywords Magnetic resonance imaging - Cerebellum . Noise $\cdot$ Resolution $\cdot$ 3D-EPI

\section{Introduction}

The cerebellum is involved in a wide range of tasks, even as simple as finger tapping, and as such is a region of interest (ROI) for a wide range of functional magnetic resonance imaging (fMRI) studies. Because of its relatively small size and fine-scale anatomical structure, compared to the cerebrum, cerebellar fMRI benefits strongly from high spatial resolution, both during acquisition $[1,2]$ and in data post-processing strategies used in fMRI [3, 4]. High spatial resolution in fMRI is most easily achieved at high field strengths, where fMRI benefits from increased signal-tonoise ratio (SNR), increased blood oxygen level-dependent (BOLD) signal $[5,6]$ and increased spatial specificity of the BOLD responses [7, 8].

Temporal signal fluctuations introduced by cardiac and respiratory processes are generally referred to as physiological noise [9]. Physiological noise contributions in fMRI data can be characterized voxel-wise in terms of the amount of explained variance introduced to the time course [10]. Cardiac related signal fluctuations are generally found close to the major vessels in the brain, while respiratory related signal fluctuations tend to increase globally in the inferior direction, because of the increasing proximity to the lungs [11]. Respiratory induced signal changes have both a motion and a susceptibility induced origin [12]. Hence, both cardiac and respiratory signal contributions are important in the 
brainstem [13]. The susceptibility changes due to respiration may, depending on the placement of the phaseencoding gradient, result in global respiration-driven distortion changes. In areas near air-water interfaces, where local field gradients exist, respiratory-induced head rotations may additionally give rise to time-varying local distortions. Physiological noise can obscure the BOLD signal of interest and many methods have been proposed to remove physiological signal components from fMRI time course data [14-17]. Importantly, physiological noise decreases in importance with increasing spatial resolution because of the larger thermal noise component [18], and acquisition of high-resolution data typically yields the application of correction strategies unnecessary [19]. On the other hand, physiological noise is also known to increase with field strength [20]. It has been shown that, at 7 Tesla, fMRI sampled with a $2 \mu \mathrm{l}$ voxel size $(1.1 \times 1.1 \times 1.8 \mathrm{~mm})$ still benefits from physiological noise removal [21].

Physiological noise contributions are also dependent on the acquisition strategy used to acquire the MR images, with segmented methods showing larger physiological noise sensitivity than single-shot approaches [22, 23]. The 3D-EPI sequence [24] is otherwise an excellent candidate for the acquisition of high-resolution fMRI data because it yields high image SNR values and offers the possibility to significantly accelerate image acquisition by application of parallel imaging in two phaseencoding directions, which provides significant flexibility in the design of an acquisition protocol [25]. Fortunately, physiological noise removal approaches tend to be more effective for 3D-EPI acquisitions than for the 2D-EPI equivalent [26, 27], yielding post-correction temporal SNR, tSNR, and BOLD sensitivity measures that are significantly improved compared to 2D-EPI. While physiological contributions differ between k-space segments, image based RETROICOR is as effective as $\mathrm{k}$-space correction strategies [28], and is generally preferable because of the data handling.

Physiological noise contributions in cerebellar fMRI are not well studied; even in whole-brain studies, the cerebellum may not be included [10, 21]. It can be hypothesized that for the cerebellum, physiological noise contributions are even more important than for the cerebrum, as it is surrounded by large arteries of the brain and in close proximity to the lungs, compared to the cerebrum. In contrast, the requirement to acquire data of high spatial resolution might yield physiological noise corrections less essential. The aim of this study was to compare physiological noise contributions in cerebellar and cerebral ROIs in high-resolution, $1 \mathrm{~mm}$ isotropic, fMRI data acquired at $7 \mathrm{~T}$ to estimate the need for physiological noise removal.

\section{Materials and methods}

All data were acquired using an actively shielded, headonly 7T MRI scanner (Siemens, Germany), equipped with a head gradient-insert and 32-channel receive coil with tight transmit sleeve (Nova Medical, Massachusetts, USA), suitable for sub-millimetre fMRI acquisitions [29].

Seven healthy volunteers participated in these experiments. Volunteers performed a visually cued finger tapping task, with $15 \mathrm{~s}$ tapping with all five fingers of one hand alternated with $15 \mathrm{~s}$ rest during a 2.5 -min functional localizer and an 8-min motor-task run. Tapping was alternated between the left and the right hand in the motortask run. In addition, $8 \mathrm{~min}$ of resting state fMRI data were acquired, during which subjects were asked to relax with their eyes open. A $0.6-\mathrm{mm}^{3}$ resolution MP2RAGE [30] data set was acquired for anatomical reference with the following parameters: matrix size: $320 \times 320 \times 256$, FOV: $192 \times 192 \times 154 \mathrm{~mm}, \mathrm{TI}_{1} / \mathrm{T}_{2}=800 / 2700 \mathrm{~ms}$, $\alpha_{1} / \alpha_{2}=4 / 11^{0} . \mathrm{TE} / \mathrm{TR} / \mathrm{TR}_{\mathrm{MP} 2 \mathrm{RAGE}}=3.03 / 7.1 / 6000 \mathrm{~ms}$, GRAPPA $=3$ and total acquisition time of $10 \mathrm{~min}$. Dielectric pads with a barium titanate suspension [31] were used to improve $B_{1}$ homogeneity over the cerebellum.

For the functional localizer, a standard multi-slice GRE-EPI sequence with sinusoidal readout was used with the following parameters: voxel size: $2.3 \mathrm{~mm}$ isotropic, matrix size $96 \times 96, \mathrm{TR} / \mathrm{TE} /$ flip angle $=2500 / 25 / 90^{\circ}$. GRAPPA $=2$ and partial Fourier 6/8 undersampling were used to obtain a $\mathrm{TE} \approx \mathrm{T}_{2}^{*}$. Forty-two near-coronal slices covering the cerebellum and centred on the motor cortex, including the basal ganglia and part of the occipital cortex, were acquired in each volume. fMRI data were analysed online to aid placement of subsequent higherresolution acquisitions. For the 1-mm fMRI acquisitions, a 3D-EPI sequence $[24,25]$ was used with voxel size $1.0 \mathrm{~mm}^{3}$ isotropic, matrix size $224 \times 224$, TR/TE/flip angle $=2520 \mathrm{~ms} / 25 \mathrm{~ms} / 17^{\circ}$. A slab of 40 near-coronal slices covering the supplementary motor area (SMA), primary sensorimotor cortex (MI/SI), secondary somatosensory cortex (SII) and the cerebellum was sampled every $2520 \mathrm{~ms}$. GRAPPA $=3$ was used to limit the length of the read-out train. The primary phase-encoding direction was approximately head-foot. For all functional and rest runs, both respiratory and cardiac data were recorded using vendor-provided hardware for physiological noise modelling, with a respiratory belt and $\mathrm{SpO} 2$ monitor, respectively. To allow synchronization of MRI data and the recorded physiological traces, the scanner volume synchronization triggers were recorded on an analog input of the physiological monitoring unit after lengthening for reliable detection.

Standard fMRI data processing, including motion correction, smoothing with a Gaussian filter of $2 \mathrm{~mm}$ FWHM, 
co-registration of the anatomical to the fMRI data, and General Linear Model (GLM) analysis, was carried out using SPM8. The basic GLM consisted of two regressors for the task-induced BOLD signal fluctuations (left hand and right hand finger tapping), modelled as a block convolved with the canonical hemodynamic response function (hrf); the motion parameters obtained from motion correction de-trended for first, second and thirrd order drifts; and three slow drift regressors. First to third order polynomials were removed from the motion parameters to reduce correlations between the two regressor sets. No temporal highpass or low-pass smoothing was applied. RETROICOR regressors up to second order were computed following [17], and additional regressors were added for the respiratory volume per unit of time (RVT) [32, 33] and the cardiac rate $(\mathrm{CR})[34,35]$. To measure the amount of data variation explained by each group of regressors, effectively comparing GLM models of different sizes, $R_{\text {adj }}^{2}$ values [34] were computed on a voxel-by-voxel basis following Eq. 1:

$R_{\mathrm{adj}}^{2}=1-\frac{N-1}{N-P-1} \frac{\sum_{i=1}^{N}\left(y_{i}-y_{i}^{\prime}\right)^{2}}{\sum_{i=1}^{N}\left(y_{i}-\bar{y}\right)^{2}}$

where $N$ is the number of volumes in the time course, $P$ is the number of regressors in the model, $y$ is the time course signal under analysis, $\bar{y}$ is the temporal average of $y$, and $y^{\prime}$ is the approximation achieved with the GLM regressors of interest. The $R_{\text {adj }}^{2}$ value provides a goodness-of-fit measure for regressors or groups of regressors that is theoretically independent from the number of degrees of freedom in the model, allowing for comparisons between different-sized models.

From the $R_{\text {adj }}^{2}$ values, the amount of variance explained, VE [27, 34], was derived following Eq. 2:

$\mathrm{VE}=100\left(R_{\mathrm{adj}}^{2}(A)-R_{\mathrm{adj}}^{2}(B)\right)$

where $R_{\mathrm{adj}}^{2}(A)$ is the result obtained with a group of regressors (for example, the motion parameters + temporal drift regressors + RETROICOR cardiac regressors) and $R_{\text {adj }}^{2}(B)$ is the results obtained with a reduced group (motion parameters + temporal drift regressors), yielding the variance explained by the difference (i.e. the RETROICOR cardiac regressors).

The motor runs were used to define ROIs in all relevant functional regions covered in the slab. For each subject, ROIs were defined in the primary sensorimotor cortex, the supplementary motor area, the secondary somatosensory cortex on the upper bank of the Sylvian fissure, cerebellar lobule $\mathrm{V}$ in the anterior lobe of the cerebellum (CV) and cerebellar lobule VIII in the posterior lobe (CVIII). The f-contrast over both left and right hand motion regressors was thresholded at $p<0.05$ familywise error corrected, leading to bilateral ROIs for all regions, apart from SMA, which is positioned medially. Voxel-specific VE values were averaged across each individual ROI.

\section{Results}

To analyse noise contributions, a GLM analysis including regressors for motor activation, slow signal drift, subject motion, respiration and heartbeat (RETROICOR), cardiac rate and respiratory volume per unit of time was performed. For all volunteers, activation was found in the bilateral primary sensory motor cortex, the supplementary motor area, bilateral secondary sensory cortex, and in the cerebellum bilaterally in lobules V and VIII. As expected, active regions and thus ROIs were largest in MI/SI and CV. ROIs contained $800 \pm 200$ voxels or $\mathrm{mm}^{3}$ in SMA (mean \pm stderr over subjects); $6000 \pm 2000$ voxels in MI/ SI, $250 \pm 50$ in SII, $2100 \pm 600$ in CV and $400 \pm 150$ in CVIII.

Figure 1 shows GLM results of the motor run for a representative subject. To visually summarise the 3D data, maximum intensity projections over the imaging slab of the f-statistic values for different regressor sets are displayed. As a reference, the slice of the anatomical image corresponding to the centre of the slab is shown in Fig. 1a. Slow drifts, or correlations with the polynomial functions, were globally distributed throughout the brain, with scattered single voxels displaying very high $f$-values (Fig. 1b). Rigid body motion yielded high signal correlations near all surfaces, especially the external brain surface (Fig. 1c), which is to be expected, as whole-brain motion leads to the largest signal changes at high-contrast edges. The cardiac RETROICOR components correlated strongly in areas of high vascular density (Fig. 1d), such as the Sylvian fissure, near the intraventricular vessels and around the superior cerebellar arteries. Focal spots of high $f$-values in Fig. 1d can be related to vessels visible in the anatomical image in Fig. 1a (white arrows). Respiratory RETROICOR components showed clearly increased $f$-values in the cerebellum compared to the cerebrum (Fig. 1e). The cardiac rate regressor (CR, Fig. 1f) yielded high $f$-values again in areas of high vascular density, though not necessarily identical to regions that displayed high $f$-values for the cardiac RETROICOR regressors (Fig. 1d). The RVT regressor (Fig. 1g) did not display any particular spatial pattern; neither for this volunteer nor for others. Finally, signal fluctuations correlating with regressors reflecting task-related BOLD signal (Fig. 1h) were found mainly in the expected ROIs and in addition in the (lateral) occipital lobe, probably arising from the visual cues appearing on the screen. Cerebral white matter was free of significant RETROICOR or CR related signal changes. 

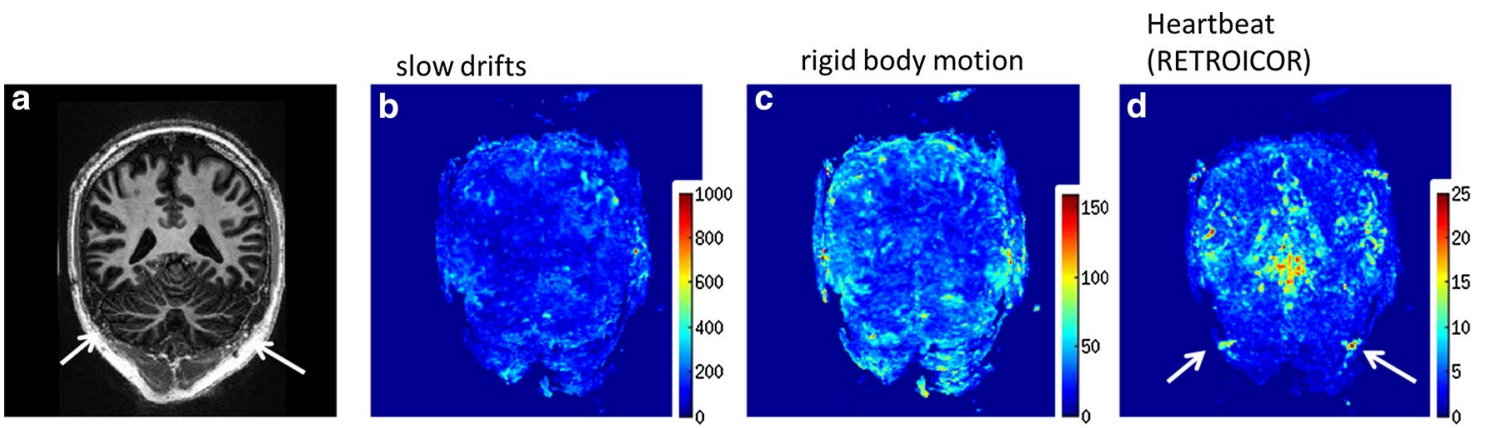

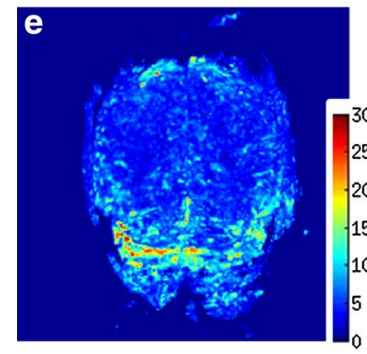

Respiration (RETROICOR)

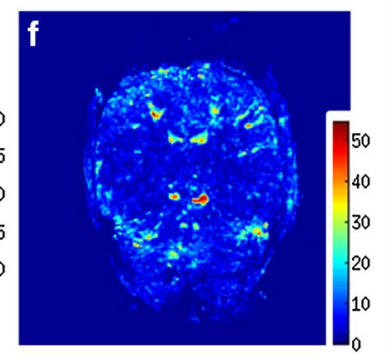

$\mathrm{CR}$

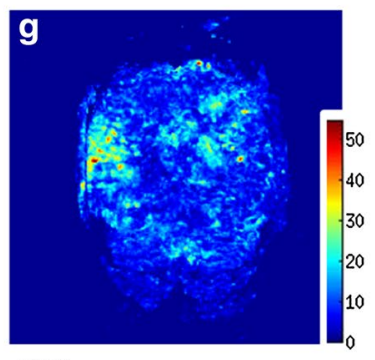

RVT

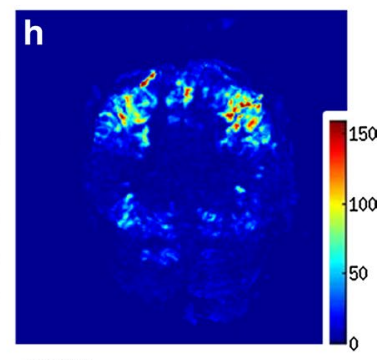

BOLD
Fig. 1 Maximum intensity projections of f-statistic values over the imaging slab for a representative subject. a Mid-slice of the anatomical MP2RAGE image. b Slow drifts explained by up to third order polynomials. c Rigid body motion as explained by motion parameters. d The four cardiac RETROICOR parameters. e The four respiratory RETROICOR parameters. f The cardiac rate regressor. $g$ The

To quantify physiological noise contributions, $R_{\text {adj }}^{2}$ maps were computed for all volunteers. $R_{\text {adj }}^{2}$ maps for a representative subject are shown in Fig. 2 for the motor task. $R_{\text {adj }}^{2}$ values were calculated for expanding models including first the slow drift regressors, modelled by first to third order polynomials, then motion parameters obtained from the six-parameter rigid body motion correction step during pre-processing, cardiac RETROICOR regressors, respiratory RETROICOR regressors, the cardiac rate regressor, the RVT regressor and finally the task-related BOLD signal, modelled with the canonical hrf. $R_{\text {adj }}^{2}$ values generally increased for each added regressor group, signifying that some signal variance was significantly explained by each of the proposed regressors. Local increases above $5 \%$, indicated with a black contour in Fig. 2, were limited to the same regions where f-statistic values for the given regressors are high. For example, inclusion of the task-induced BOLD signal regressors leads to $>5 \%$ changes in MI/SI, SMA, CV and CVIII for the slice shown (See white arrows in Fig. 2h).

$R_{\text {adj }}^{2}$ values were averaged over the ROIs and the average explained variance per regressor group was calculated (Tables 1, 2). Explained variance contributions from cardiac RETROICOR components were significant ( $t$ tests, $p<0.05$ ) in all ROIs in both rest and motor runs (see * respiratory volume per unit of time regressor. $\mathbf{h}$ Task-induced BOLD signal according to modelled hrf. White arrows indicate the position of large vessels in the anatomical slice (a) and in the cardiac RETROICOR f-statistic maximum intensity projection (d). Note the different scaling factors for each f-statistic MIP

in Tables 1 and 2). Respiratory RETROICOR components were significant in all ROIs in the motor run, and in all but the MI/SI ROI for the rest run, due to a single outlier. Cardiac rate signal fluctuations were significant in CVIII, MI/ SI and SMA in the rest run and in CVIII, CV, SII and MI/SI in the motor run. RVT contributions were significant in all ROIs in the rest run, but only in $\mathrm{CV}$ and MI/SI in the motor run. To visualise the results, $R_{\text {adj }}^{2}$ values are also shown in pie charts: explained variance in the rest runs is displayed in the top row of Fig. 3, while the bottom row displays results from the motor run.

Slow drifts accounted for a large part of the variance for all ROIs and run types, 25-30\% in the rest runs and 20-25\% in the motor runs. Motion parameters further accounted for large percentages of variance: ranging from 12 to $18 \%$ in the rest runs and 11 to $20 \%$ in the motor runs. Cardiac RETROICOR regressors explained 0.9$1.6 \%$ of variance, with the largest amount of explained variance found in the rest run in SII (Table 1 and middle panels Fig. 3). Respiratory RETROICOR regressors increased for more inferior ROIs, up to $3 \%$ in the CVIII ROI during the rest run and $2.4 \%$ in the motor run, while down to 1.3 and $1.4 \%$ for the motor run in MI/SI and SMA ROIs. This significant increase $(80 \%, t$ test $p<0.05)$ of respiratory $\mathrm{EV}$ for the cerebellum, relative to the superior 


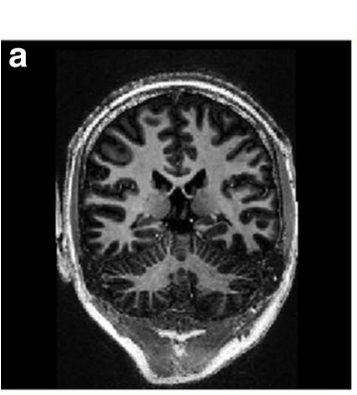

slow drifts
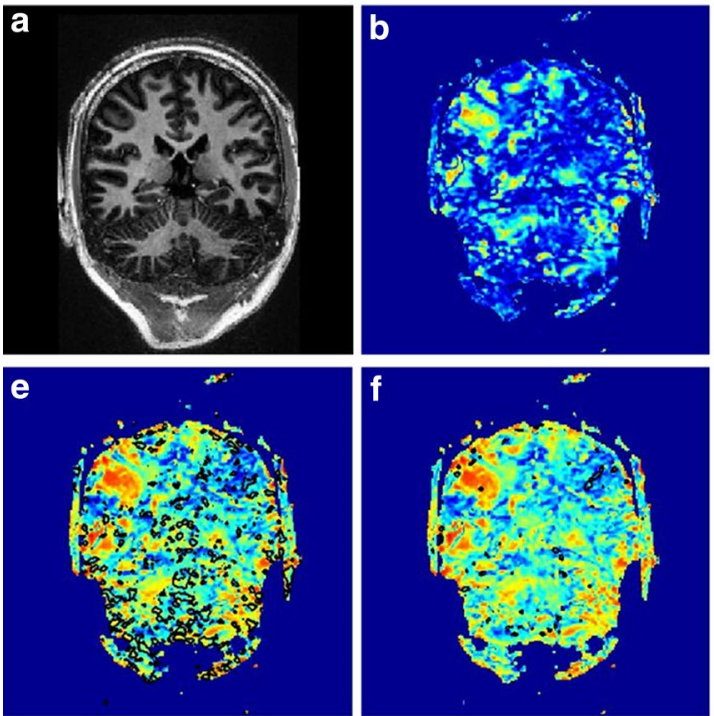

Respiration (RETROICOR)

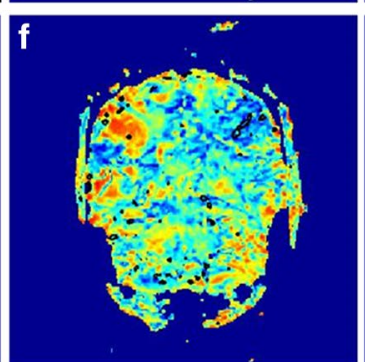

CR rigid body motion
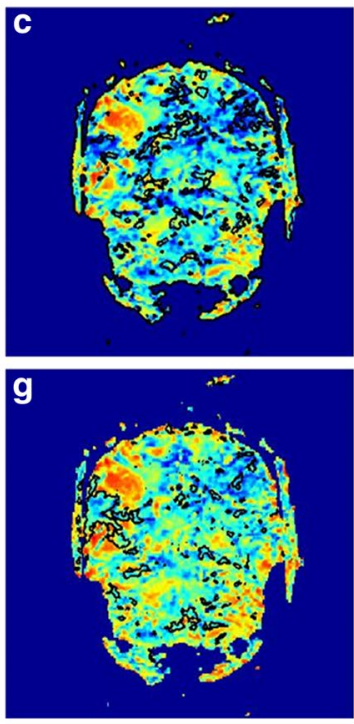

RVT
Heartbeat

(RETROICOR)
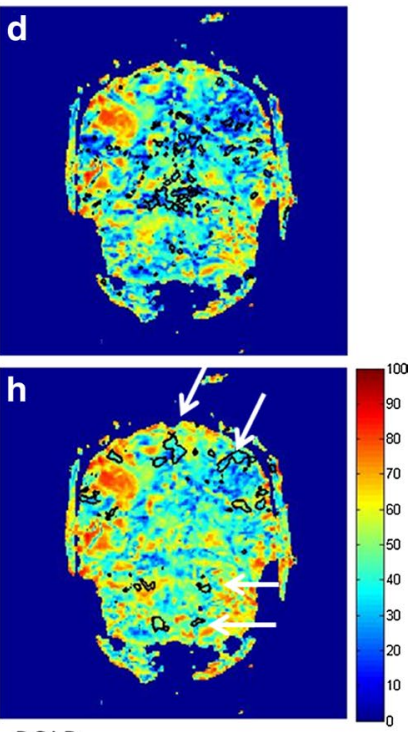

BOLD

Fig. $2 R_{\text {adj }}^{2}$ maps for a representative subject (same subject as in Fig. 1). All maps are displayed using the same color scale, and the same slice, positioned near the middle of the imaging slab, is displayed in all panels. Black contours indicate regions with more than $5 \%$ change from the model not including the last added regressor group. a Corresponding slice of the anatomical MP2RAGE image. b First to third order polynomials. c Six-Parameter rigid body motion correction. d Four Cardiac RETROICOR regressors. e Four Respiratory RETROICOR regressors. f CR. g RVT. $\mathbf{h}$ Task-induced BOLD signal changes. White arrows indicate the positions of SMA, M1/S1, $\mathrm{CV}$ and CVIII
Table 1 Average and standard error of explained variance per $\mathrm{ROI}$ in resting state runs

\begin{tabular}{lrrrrr}
\hline EVIROI & \multicolumn{1}{l}{ CVIII } & \multicolumn{1}{l}{ CV } & \multicolumn{1}{l}{ SII } & \multicolumn{1}{l}{ M1/S1 } & \multicolumn{1}{c}{ SMA } \\
\hline Slow drift & $30.5 \pm 4.9^{*}$ & $28.0 \pm 4.1^{*}$ & $24.3 \pm 5.5^{*}$ & $30.5 \pm 4.1^{*}$ & $25.0 \pm 4.1^{*}$ \\
Motion & $18.3 \pm 2.5^{*}$ & $16.3 \pm 2.2^{*}$ & $12.6 \pm 1.8^{*}$ & $15.7 \pm 2.0^{*}$ & $15.3 \pm 2.3^{*}$ \\
RETROcard & $0.9 \pm 0.2^{*}$ & $1.1 \pm 0.2^{*}$ & $1.6 \pm 0.5^{*}$ & $1.1 \pm 0.2^{*}$ & $1.5 \pm 0.3^{*}$ \\
RETROresp & $2.9 \pm 0.5^{*}$ & $2.6 \pm 0.4^{*}$ & $2.7 \pm 0.7^{*}$ & $2.8 \pm 1.2$ & $2.3 \pm 0.7^{*}$ \\
CR & $0.8 \pm 0.3^{*}$ & $1.2 \pm 0.7$ & $2.3 \pm 1.4$ & $1.1 \pm 0.4^{*}$ & $0.6 \pm 0.2^{*}$ \\
RVT & $0.4 \pm 0.1^{*}$ & $1.0 \pm 0.2^{*}$ & $1.3 \pm 0.2^{*}$ & $0.9 \pm 0.3^{*}$ & $1.3 \pm 0.4^{*}$ \\
Other & $46.1 \pm 3.1^{*}$ & $49.8 \pm 2.0^{*}$ & $55.2 \pm 5.9^{*}$ & $47.9 \pm 5.0^{*}$ & $54.1 \pm 5.1^{*}$ \\
\hline
\end{tabular}

Explained variance values in the resting state run. Values are presented as the mean over subjects \pm standard error. All ROIs were bilateral, apart from SMA. See also Fig. 3, top row

* Significant explained variance contributions $(t$ test $p<0.05)$
ROIs was found in the motor run, while intra-subject differences in the rest run meant no significant differences were found between ROIs for the respiratory RETROICOR components. The $\mathrm{CR}$ regressors explained approximately $1 \%$ of variance, comparable to previously reported values [34]. The RVT regressor explained approximately $1 \%$ of variance in the rest run for all ROIs, but less in the motor runs, ranging from $0.2 \%$ in SMA to $0.9 \%$ in CVIII.

Task-induced BOLD signal fluctuations, as modelled with the canonical hrf, accounted for 9-20\% of variance, with large differences between ROIs. In the MI/SI and CV ROIs, a much larger percentage of signal fluctuations was attributed to the task than in SII or CVIII. For all ROIs, the percentage of variance explained by the task-induced BOLD signal was larger than the sum of the variance explained by the physiological regressors.

For both rest and motor runs, the largest category is formed by the 'other' sources, which include all signal variance not explained by any regressors in the model, such as the thermal noise, system instabilities and BOLD 
Table 2 Average and standard error of explained variance per ROI in motor task runs

\begin{tabular}{lccccc}
\hline EVIROI & CVIII & CV & SII & M1/S1 & SMA \\
\hline Slow drift & $23.1 \pm 5.6^{*}$ & $21.1 \pm 3.6^{*}$ & $20.1 \pm 1.4^{*}$ & $21.4 \pm 2.6^{*}$ & $21.3 \pm 3.4^{*}$ \\
Motion & $19.6 \pm 6.8^{*}$ & $14.4 \pm 4.0^{*}$ & $11.4 \pm 0.8^{*}$ & $12.7 \pm 1.1^{*}$ & $11.2 \pm 2.0^{*}$ \\
RETROcard & $1.0 \pm 0.2^{*}$ & $1.0 \pm 0.2^{*}$ & $1.4 \pm 0.3^{*}$ & $0.8 \pm 0.0^{*}$ & $1.0 \pm 0.2^{*}$ \\
RETROresp & $2.4 \pm 0.6^{*}$ & $2.0 \pm 0.3^{*}$ & $1.9 \pm 0.2^{*}$ & $1.3 \pm 0.2^{*}$ & $1.4 \pm 0.3^{*}$ \\
CR & $0.7 \pm 0.2^{*}$ & $0.5 \pm 0.1^{*}$ & $0.7 \pm 0.3$ & $0.9 \pm 0.3^{*}$ & $1.4 \pm 0.6$ \\
RVT & $0.9 \pm 0.5$ & $0.7 \pm 0.2^{*}$ & $0.6 \pm 0.6$ & $0.5 \pm 0.1^{*}$ & $0.2 \pm 0.6$ \\
BOLD & $9.0 \pm 2.1^{*}$ & $13.4 \pm 1.3^{*}$ & $10.4 \pm 0.9^{*}$ & $17.6 \pm 1.6^{*}$ & $11.3 \pm 0.8^{*}$ \\
Other & $43.3 \pm 6.3^{*}$ & $46.9 \pm 3.5^{*}$ & $53.4 \pm 1.0^{*}$ & $45.0 \pm 2.0^{*}$ & $52.7 \pm 3.3^{*}$ \\
\hline
\end{tabular}

Explained variance values in the motor task run. Values are presented as the mean over subjects \pm standard error. All ROIs were bilateral, apart from SMA. See also Fig. 3, bottom row
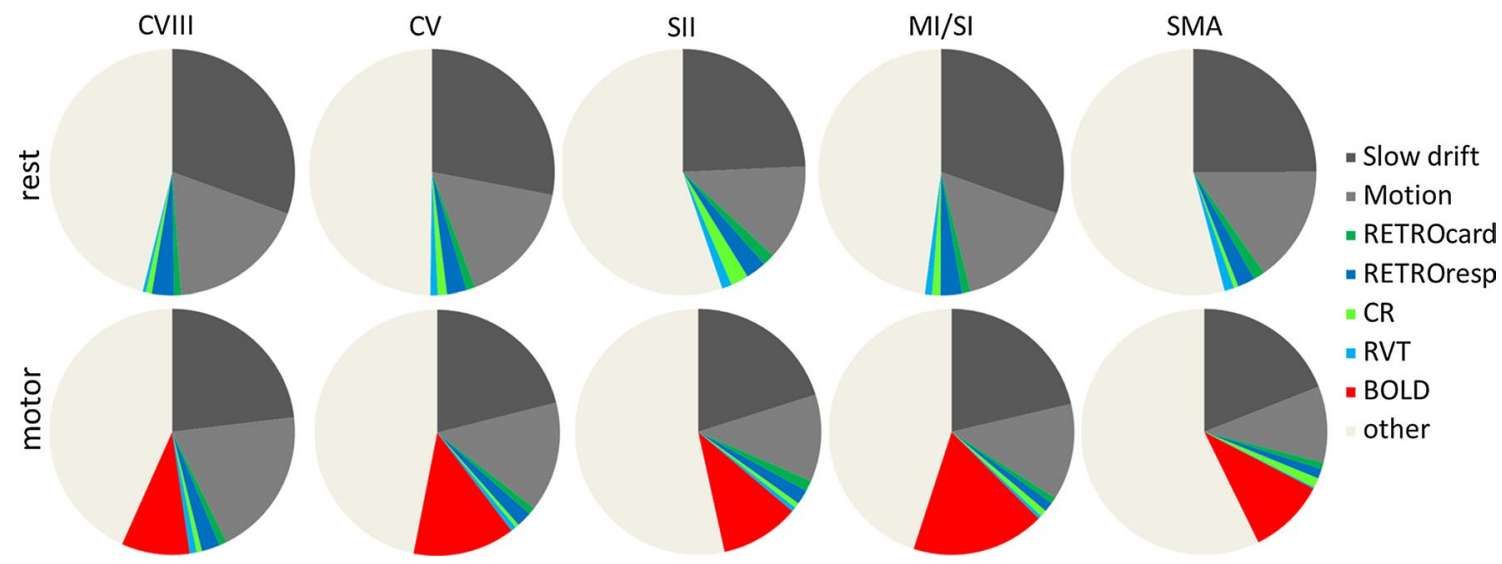

Fig. 3 Pie charts with explained variance per ROI, averaged over all subjects. For all ROIs, 'other' sources (thermal noise, non-task related BOLD-induced signal fluctuations, system instabilities, etc.) formed the largest contribution. Respiratory related components were increased in the inferior ROIs, while cardiac related regressors were highest in SII. CVIII cerebellar lobule VIII, CV cerebellar lobule V, SII secondary somatosensory cortex, MI/SI primary sensorymotor cortex, SMA supplementary motor area signal arising from ongoing brain processes. Some residual physiological noise, not explained by the included physiological regressors, might also exist. In the case of the motor run, the 'other sources' category also includes BOLD signal fluctuations not explained by the canonical hrf, though these are likely to be small, given the large amount of variance explained by the task-related BOLD regressors.

No significant differences in numbers of voxels determined significantly active and/or maximum t-score per ROI were found in these data when comparing the results of GLMs with only the task-related regressors, motion parameters and slow drift regressors versus the full model including all RETROICOR components, CR and RVT. While a non-significant increase of $23 \pm 20 \%$ in number of active voxels was found in the cerebellar lobule VIII, for all other ROIs, the standard error was larger than the mean difference between analysis with and without inclusion of physiological noise regressors in the GLM.

\section{Discussion}

These data show that physiological noise contributions in fMRI data are still significant at 1-mm spatial resolution (Tables 1,2). The choice of acquisition sequence and the associated parameter settings influences the amount of physiological noise to be expected in the fMRI data $[18,21,23,27]$. Here, data were acquired at $7 \mathrm{~T}$, which is expected to yield larger physiological noise contributions than lower field strengths [20], and while a relatively small voxel size was used, which reduces the relative contribution of physiological signal components [18, 21], data were acquired with a 3D-EPI sequence, which is more sensitive to physiological noise than its 2D counterpart [23, 27]. The choice for the use of the 3D-EPI sequence was motivated by the possibility to acquire even higher spatial resolution data; a constant goal for ultra-high field fMRI.

The distribution of explained variance per regressor group in the different ROIs shows ROI-typical behaviour, which can mostly be explained by the anatomical position 
of the ROI in the brain. Taking a closer look at the ROIs (Fig. 3) in inferior-to-superior order, CVIII clearly has a larger contribution from the RETROICOR respiratory regressors than the other ROIs, due to its relative proximity to the lungs. Task-induced BOLD explains relatively little variance, perhaps because of the limited size of the ROI and resulting partial volume effects from surrounding tissue. BOLD-based activation also tends to be smaller in lobule VIII than in lobule V [36]. Motion parameters also accounted for a larger part of the variance in CVIII than in the more superior ROIs, probably because the positioning of the subjects' head in the rf-coil most easily allows a rotation around the $x$ axis (pitch), which most affects the inferior brain regions. The variance pattern in $\mathrm{CV}$ is similar to that in CVIII, albeit with a larger contribution of taskrelated BOLD in the motor run, which is probably due to the larger size of the ROI and reduced partial volume effects resulting from that.

The secondary somatosensory cortex ROI tends to show the largest amount of variance explained by the cardiacrelated regressors (CR and cardiac RETROICOR) due to the presence of the large vessels that lie in the Sylvian fissure, although these differences were not statistically significant. The MI/SI ROI is, during the motor run, dominated by task-related BOLD signal, and finally the SMA ROI has relatively modest contributions from all factors of interest here, apart from a cardiac RETROICOR contribution, which is relatively large compared to the other ROIs. This is probably related to the presence of the large vessels in the interhemispheric fissure, which might be included in the SMA ROI.

For all ROIs apart from MI/SI, the sum of all physiological contributions (RETROICOR, CR and RVT) is approximately equal to half that of the task-induced BOLD signal. A reduction of the variance by such an amount would be expected to result in significant increases in BOLD sensitivity. Such increases have indeed been demonstrated [21, 27], although in those studies, larger voxel sizes than in the current study were used. However, reductions in positive correlations after physiological noise removal have also been reported [16]. In this study, no significant differences in either spatial extent of activation or maximum z-score were found, apart from a non-significant difference for the ROI in cerebellar lobule VIII. The difference in BOLD sensitivity would be expected to be largest in ROIs with a relatively large contribution from physiological noise and small task-induced BOLD signal, such as cerebellar lobule VIII. Removal of physiological noise is commonly applied to resting state fMRI, but this is less common in task-based fMRI experiments. The variance distributions shown here, which demonstrate significant percentages of variance explained by physiological regressors, in both rest (Table 1) and motor task (Table 2) runs, indicate that, even though no increase in BOLD sensitivity was found for this particular task, the use of some physiological noise correction scheme is also highly recommended for task-based fMRI to reduce non-task related temporal signal variations, making the GLM statistical analyses more accurate.

The removal of physiological noise can be relatively easily performed via RETROICOR if cardiac and respiratory recordings are available. If these are not acquired at the time of scanning, or are not of sufficient quality, alternative methods also exist. For example, the inclusion of the average white matter and/or CSF signal time course as a regressor of no interest [16] can help to reduce variance of no interest without requiring the introduction of additional monitoring into the experimental setup. Another promising method is the use of signal from a reference region, which can be defined anatomically [37] or via a separately acquired resting state fMRI run [38]. This method has been shown to be very effective in improving the BOLD sensitivity of 3D-EPI fMRI data [27], but it does not allow the characterization of the noise distribution.

\section{Conclusion}

Significant correlations with both cardiac and respiratory RETROICOR components were found in all healthy volunteer data, even at the high spatial resolution used $(1 \mathrm{~mm}$ isotropic), with higher respiratory-induced signal fluctuations in the cerebellum than in the forebrain. Because of the increased respiratory-induced signal fluctuations, physiological noise correction of fMRI data is especially important in the cerebellum.

Acknowledgments This work was supported by Centre d'Imagerie BioMédicale (CIBM) of the UNIL, UNIGE, HUG, CHUV, EPFL, and the Leenaards and Jeantet Foundations, the Portuguese Science Foundation (FCT) through grant SFRH/BD/51449/2011 to JJ and by a project grant of the Swiss National Science Foundation (31003A_153070) to WvdZ.

Conflict of interest The authors declare that they have no conflict of interest.

Ethical standard All human and animal studies have been approved by the appropriate ethics committee and have therefore been performed in accordance with the ethical standards laid down in the 1964 Declaration of Helsinki and its later amendments. All volunteers provided written informed consent prior to their participation.

\section{References}

1. Marques JP, van der Zwaag W, Granziera C, Krueger G, Gruetter R (2010) Cerebellar cortical layers: in vivo visualization with structural high-field-strength MR imaging. Radiology 254:942-948 
2. Van der Zwaag W, Kusters R, Magill A, Gruetter R, Martuzzi R, Blanke O, Marques JP (2013) Digit somatotopy in the human cerebellum: a 7T fMRI study. NeuroImage 67:354-362

3. Diedrichsen J, Maderwald S, Küper M, Thürling M, Rabe K, Gizewski ER, Ladd ME, Timmann D (2011) Imaging the deep cerebellar nuclei: a probabilistic atlas and normalization procedure. NeuroImage 54:1786-1794

4. Küper M, Wünnemann MJS, Thürling M, Stefanescu RM, Maderwald S, Elles HG, Göricke S, Ladd ME, Timmann D (2013) Activation of the cerebellar cortex and the dentate nucleus in a prism adaptation fMRI study. Hum Brain Mapp. doi:10.1002/ hbm. 22274

5. Yacoub E, Shmuel A, Pfeuffer J, Van De Moortele PF, Adriany G, Andersen P, Vaughan JT, Merkle H, Ugurbil K, Hu X (2001) Imaging brain function in humans at 7 Tesla. Magn Reson Med 45:588-594

6. Gizewski ER, de Greiff A, Maderwald S, Timmann D, Forsting M, Ladd ME (2007) fMRI at 7 T: whole-brain coverage and signal advantages even infratentorially? NeuroImage 37:761-768

7. Van der Zwaag W, Francis S, Head K, Peters A, Gowland P, Morris P, Bowtell R (2009) fMRI at 1.5, 3 and 7 T: characterising BOLD signal changes. NeuroImage 47:1425-1434

8. Francis S, Panchuelo RS (2014) Physiological measurements using ultra-high field fMRI: a review. Physiol Meas 35:R167-R185

9. Krüger G, Kastrup A, Glover GH (2001) Neuroimaging at $1.5 \mathrm{~T}$ and $3.0 \mathrm{~T}$ : comparison of oxygenation-sensitive magnetic resonance imaging. Magn Reson Med 45:595-604

10. Bianciardi M, Fukunaga M, van Gelderen P, Horovitz SG, de Zwart JA, Shmueli K, Duyn JH (2009) Sources of functional magnetic resonance imaging signal fluctuations in the human brain at rest: a $7 \mathrm{~T}$ study. Magn Reson Imaging 27:1019-1029

11. Van de Moortele P-F, Pfeuffer J, Glover GH, Ugurbil K, Hu X (2002) Respiration-induced B0 fluctuations and their spatial distribution in the human brain at 7 Tesla. Magn Reson Med 47:888-895

12. Raj D, Paley DP, Anderson AW, Kennan RP, Gore JC (2000) A model for susceptibility artefacts from respiration in functional echo-planar magnetic resonance imaging. Phys Med Biol 45:3809-3820

13. Brooks JCWP, Faull OK, Pattinson KTS, Jenkinson MP (2013) Physiological noise in brainstem fMRI. Front Hum Neurosci 7:623

14. Hagberg GE, Bianciardi M, Brainovich V, Cassara AM, Maraviglia B (2012) Phase stability in fMRI time series: effect of noise regression, off-resonance correction and spatial filtering techniques. NeuroImage 59:3748-3761

15. Thomas CG, Harshman RA, Menon RS (2002) Noise reduction in BOLD-based fMRI using component analysis. NeuroImage 17:1521-1537

16. Chang C, Glover GH (2009) Effects of model-based physiological noise correction on default mode network anti-correlations and correlations. NeuroImage 47:1448-1459

17. Glover GH, Li TQ, Ress D (2000) Image-based method for retrospective correction of physiological motion effects in fMRI: RETROICOR. Magn Reson Med 44:162-167

18. Bodurka J, Ye F, Petridou N, Murphy K, Bandettini PA (2007) Mapping the MRI voxel volume in which thermal noise matches physiological noise-Implications for fMRI. NeuroImage 34:542-549

19. Triantafyllou C, Hoge RD, Wald LL (2006) Effect of spatial smoothing on physiological noise in high-resolution fMRI. NeuroImage 32:551-557
20. Triantafyllou C, Hoge RD, Krueger G, Wiggins CJ, Potthast A, Wiggins GC, Wald LL (2005) Comparison of physiological noise at $1.5 \mathrm{~T}, 3 \mathrm{~T}$ and $7 \mathrm{~T}$ and optimization of fMRI acquisition parameters. NeuroImage 26:243-250

21. Hutton C, Josephs O, Stadler J, Featherstone E, Reid A, Speck O, Bernarding J, Weiskopf N (2011) The impact of physiological noise correction on fMRI at $7 \mathrm{~T}$. NeuroImage 57:101-112

22. Lai S, Glover GH (1998) Three-dimensional spiral fMRI technique: a comparison with 2D spiral acquisition. Magn Reson Med 39:68-78

23. Van der Zwaag W, Marques JP, Kober T, Glover G, Gruetter R, Krueger G (2012) Temporal SNR characteristics in segmented 3D-EPI at 7T. Magn Reson Med 67:344-352

24. Poser BA, Koopmans PJ, Witzel T, Wald LL, Barth M (2010) Three dimensional echo-planar imaging at 7 Tesla. NeuroImage 51:261-266

25. Narsude M, van der Zwaag W, Kober T, Gruetter R, Marques JP (2014) Improved temporal resolution for functional studies with reduced number of segments with three-dimensional echo planar imaging. Magn Reson Med 72:786-792

26. Lutti A, Thomas DL, Hutton C, Weiskopf N (2013) High-resolution functional MRI at $3 \mathrm{~T}$ : 3D/2D echo-planar imaging with optimized physiological noise correction. Magn Reson Med 69:1657-1664

27. Jorge J, Figueiredo P, van der Zwaag W, Marques JP (2013) Signal fluctuations in fMRI data acquired with 2D-EPI and 3D-EPI at 7 Tesla. Magn Reson Imaging 31:212-220

28. Tijssen RHN, Jenkinson M, Brooks JCW, Jezzard P, Miller KL (2014) Optimizing RetroICor and RetroKCor corrections for multi-shot 3D FMRI acquisitions. NeuroImage 84:394-405

29. Salomon R, Darulova J, Narsude M, van der Zwaag W (2014) Comparison of an 8-channel and a 32-channel coil for high-resolution FMRI at 7 T. Brain Topogr 27:209-212

30. Marques JP, Kober T, Krueger G, van der Zwaag W, Van de Moortele P-F, Gruetter R (2010) MP2RAGE, a self bias-field corrected sequence for improved segmentation and T1-mapping at high field. NeuroImage 49:1271-1281

31. Teeuwisse WM, Brink WM, Webb AG (2012) Quantitative assessment of the effects of high-permittivity pads in 7 Tesla MRI of the brain. Magn Reson Med 67:1285-1293

32. Birn RM, Diamond JB, Smith MA, Bandettini PA (2006) Separating respiratory-variation-related fluctuations from neuronalactivity-related fluctuations in fMRI. NeuroImage 31:1536-1548

33. Birn RM, Smith MA, Jones TB, Bandettini PA (2008) The respiration response function: the temporal dynamics of fMRI signal fluctuations related to changes in respiration. NeuroImage 40:644-654

34. Shmueli K, van Gelderen P, de Zwart JA, Horovitz SG, Fukunaga M, Jansma JM, Duyn JH (2007) Low-frequency fluctuations in the cardiac rate as a source of variance in the resting-state fMRI BOLD signal. NeuroImage 38:306-320

35. Chang C, Cunningham JP, Glover GH (2009) Influence of heart rate on the BOLD signal: the cardiac response function. NeuroImage 44:857-869

36. Küper M, Dimitrova A, Thürling M, Maderwald S, Roths J, Elles HG, Gizewski ER, Ladd ME, Diedrichsen J, Timmann D (2011) Evidence for a motor and a non-motor domain in the human dentate nucleus-an fMRI study. NeuroImage 54:2612-2622

37. De Zwart JA, Van Gelderen P, Fukunaga M, Duyn JH (2008) Reducing correlated noise in fMRI data. Magn Reson Med 59:939-945

38. Bianciardi M, van Gelderen P, Duyn JH, Fukunaga M, de Zwart JA (2009) Making the most of fMRI at 7 T by suppressing spontaneous signal fluctuations. NeuroImage 44:448-454 